\title{
NON-NECROTIZING INFLAMMATORY GRANULOMAS (N-NGS) IN THE COURSE OF INFLAMMATORY BOWEL DISEASE - IMMUNOLOGY AND CLINICAL MANIFESTATION OF INTESTINAL AND RESPIRATORY N-NGS
}

DOI: 10.36740/WLek202007143

\author{
Igor Rakoczy, Marzena Ocetek, Marzena Wiatr, Patrycja Zięba, Michał Panek, Piotr Kuna \\ DEPARTMENT OF INTERNAL MEDICINE, ASTHMA AND ALLERGY, MEDICAL UNIVERSITY OF LODZ, LODZ, POLAND
}

\begin{abstract}
The aim:The article describes and summarizes the immunological pathomechanisms controlling the development of non-necrotizing granulomas in the course of non-specific inflammatory bowel diseases (IBD) in lungs and intestines; it also reviews the possible clinical correlations between the processes in the gastrointestinal and respiratory tracts based on the example of Crohn's disease (CD) and non-specific inflammatory bowel disease (IBC).

While the dominant cell subpopulation in ulcerative colitis (UC) is Th2, which produces interleukins IL-4, IL-5, IL-6, IL-10 and IL-13 and Th17 cells; CD characterized by the Th1 cell subpopulation and macrophages predominate, producing IL-23. These are considered to be the key factors crucial for the occurrence of chronic inflammation. Another important causative factor of non-specific inflammatory bowel diseases and granulation is the expression of CD40/CD40L proteins on activated T-cells, i.e. type 2 transmembrane proteins similar to TNF-alpha. However, the interactions between gastrointestinal neuroendocrine peptides/amines (NEPA) and the immune system are believed to have a significant influence on the pathophysiology of non-specific inflammatory bowel diseases and non-necrotizing granulation. The key functions of the immune response of the gastrointestinal tract are managed by the neuroendocrine regulatory system (NES) whose activities govern the production of various hormones including chromogranin/secretogranin, serotonin, vasoactive intestinal polypeptide (VIP), neuropeptide Y (NPY), substance P, somatostatin or ghrelin.
\end{abstract}

KEY WORDS: Crohn's disease, inflammatory bowel diseases (IBD), non-necrotizing granulomas (N-NGs), ulcerative colitis (UC)

Wiad Lek. 2020;73(7):1545-1553

\section{INTRODUCTION}

Although the etiology of non-specific bowel inflammation remains unclear, it is known to involve persistent bowel infections, and mucosal barrier defects, as well as various immunological, genetic and environmental factors. It has been found that non-necrotizing granulomas commonly occur in the intestines and the lungs as characteristic lesions during the course of non-specific inflammatory bowel diseases. Immune system dysfunction and mucous membrane disorders play a significant role in this type of granulation: one aspect of the chronic inflammation underlying non-specific inflammatory bowel diseases (IBD).

Non-specific IBD is a term defining a group of inflammatory conditions of the gastrointestinal tract. The two most common, and most frequently described, conditions representing this group are non-specific ulcerative colitis (UC) and Crohn's disease (CD). Although the precise cause remains unknown, the etiology of the diseases is conditioned by genetic and environmental factors, as well as the immune system of the patient $[1,2]$.

It is estimated that 1 to 1.5 million Americans and 2.5 to 3.0 million Europeans suffer from non-specific IBD, as do hundreds of thousands of individuals around the rest of the world. UC is more widespread than $\mathrm{CD}$ [3] with the ratio of $\mathrm{UC}$ to $\mathrm{CD}$ being found to be as high as 15:1, identified in Poland in 2005 [4].
The annual incidence rates of UC per 100000 individuals vary worldwide: from 6.3 in Asia and the Middle East, through 19.2 in North America to 24.3 in Europe. Similarly, the incidence of CD ranges from 5.0 per 100000 individuals in Asia and the Middle East, to 12.7 in Europe and 20.2 in North America [5]. In Poland non-specific IBDs affect about $0.1 \%$ of the population; however, the number is increasing [6] One study indicates the overall annual incidence to be 2.7 per 100000 children in Poland [7].

Non-specific IBD occurs most frequently in white populations; however, its incidence has grown in other ethnic groups $[8,9]$. Considerable variation exists between populations regarding its occurrence: for example, IBD occurrence is three times higher in the Jewish population than in some other non-Jewish populations [8].

\section{THE AIM}

Recent studies have revealed a relationship between the occurrence of $\mathrm{CD}$ and the presence of mutations in such genes as ATG16L1, CX3CR1, IL-23R and MMP3. Additionally, blood serum-producing cells, growth factors, miRNA and microbiological antibodies are also associated with lesions in patients with $\mathrm{CD}[10]$. 


\section{REVIEW AND DISSUSION}

\section{ETHIOPATHOGENESIS}

The etiology of non-specific IBD remains unknown. The disease process involves persistent bowel infections and mucosal barrier defects, as well as immunological, genetic and environmental factors [11].

Family inheritance suggests that the disease might have a genetic basis. Indeed, several genes indicating susceptibility to the disease have been identified, as well as 163 positions of nucleotide polymorphisms in the genome. Susceptibility to the disease depends on the mutation of the NOD2 protein gene, CARD15, located on chromosome 16. The gene helps maintain the balance between the intestinal microbiota and the host immune response to inflammation by activating a pro-inflammatory transcription program; this system participates in the production of pro-inflammatory proteins and increases the intensity of the inflammatory response and other immunology pathways, inter alia by regulation of macrophage activation in response to bacterial liposaccharides [12]. The presence of two alleles of the mutated NOD2 gene increases the risk of developing CD by even 20-40 times [2].

Patients presenting with non-specific IBD also display differences in the quality and amount of their bacterial flora. A crucial role in the development of inflammation may be played by certain strains of Escherichia coli and Bacteroides vulgatus [1].

Smoking reduces both the risk of occurrence and the severity of $\mathrm{UC}$, - however, it also increases the risk of $\mathrm{CD}$ and worsens its course $[1,2]$.

A significant factor in the pathogenesis of non-specific IBD is dysfunction of the immune system of the mucous membrane. It is believed that out of the various inflammatory cells of the intestines, mucosal T CD4 + cells play a central role in both the induction and maintenance of chronic inflammation through production of pro-inflammatory cytokines. While in physiological conditions, the lamina priopria of the intestinal mucosa contains large numbers of various immune cells, such as T, B, NK, NKT cells, dendritic cells, macrophages, mastocytes, neutrophils, eosinophils and stromal cells (fibroblasts), in the case of inflammation, a large number of activated immune cells are able to penetrate the intestinal mucosa. These cells produce numerous inflammatory mediators; in addition, fibroblasts and the endothelial cells of intestinal mucosa vessels also present high levels of chemokines, selectins (e.g. E selectins) and intracellular adhesion molecule-1 (ICAM-1 or CD54). These factors interact with the leukocytes circulating in the blood, making them migrate to the intestinal mucosa and promoting a local inflammatory response [13].

The courses both of UC and CD are characterized by increased activation of T CD4+ (Th) lymphocytes. Activated $\mathrm{T}$ lymphocytes produce increased amounts of cytokines, which further progress the course of the immune response [1]. In patients suffering from $\mathrm{CD}, \mathrm{CD} 4+$ lymphocytes are able to produce large amounts of pro-inflammatory cytokines bound to Th1 / Th17 (e.g. interferon-gamma - IFN- $\gamma$, tumour necrosis factor - TNF and interleukin 17A - IL-17A) [13].

In contrast, in patients with UC, the Th2 subpopulation dominates. These cells produce interleukins IL-4, IL-5, IL6, IL-10 and IL-13; they also produce Th17 cells, which secrete various interleukins including IL-17A. An imbalance between the levels of pro-inflammatory cytokines, such as IL-12, IL-18, IL-21 and IL-23, and anti-inflammatory cytokines, such as TGF- $\beta$, IL-10, IL-25, IL-33 and IL-37, results in inflammation of the intestinal mucosa [13]. Although it was previously believed that IL-12 was the key factor initiating the inflammatory response by Th1 in CD, more recent studies in 2006 suggest that it may be IL-23 [14][15].

UC itself is triggered by a Th2-like response, the main product of which being natural killer (NK) cells which generate IL-5 and IL-13 [16].

During mucosal inflammation in patients with non-specific IBD, the titre of regulatory lymphocytes (Foxp3), T-cells (Treg) and FoxP3-IL-10+ CD4+ decreases. This results in part of the intestinal mucosa displaying decreased immunity, as well as increased support for the local immune response, leading to damage to the intestinal mucosa [13].

IL-22 is a member of the IL-2 cytokine family, which is produced mainly by $\mathrm{CD} 4+\mathrm{T}$ - lymphocytes. It shows a pleiotropic ability to regulate differentiation and functions of T-cells and strengthens antigen-specific clonal expansion. IL-21 is also associated with the immune response through Th2 and plays a role in inhibiting the differentiation of naive Th-cells into Th1 cells producing IFN. It promotes the maturation and activation of human NK cells, triggers other biological functions in B-lymphocytes and regulates the differentiation and production of antibodies.

Elevated amounts of IL-21 are observed in the intestines of patients with non-specific IBD. In addition, it increases the expression of transcription factors bound to Thl and IFN- $\gamma$ in T and NK cells and enhances the cytotoxic activity of NK cells. IL-21 also inhibits peripheral Treg differentiation and makes CD4+ T-lymphocytes resistant to immune suppression by Treg [13]. IL-13 is an additional pathway for the production of pathogenic Th-17 cells. The combination of TGF-beta and IL-21 also inhibits Foxp3 expression in naive T cells via IL-17 induction, which suggests that IL-21 is able to regulate the mutual pathway of development of Treg and Th17 cell generation [17].

Therefore, IL-21 may damage the intestines via a range of signalling pathways, and its neutralization may be of value in the treatment of non-specific IBD.

IL-23, produced mainly by macrophages, is also known to be one of the key cytokines in non-specific IBD and to play an important role in promoting chronic bowel inflammation.

Overexpression of IL-23 in the intestinal mucosa inhibits the production of IL-10 [18][13]. IL-10 induces the Th-2 response; this plays a crucial role in adaptive immunity by inducing the production of specific antibodies to eliminate invading microorganisms and absorb their products [13]. 
IL-10 is one of the most effective cytokines responsible for the regulation of the immunological system. Its reduced expression in non-specific IBD weakens the host defence by decreasing the production of IgA in the intestines [18].

Both Th17 lymphocytes and the cytokines they secrete, for example IL-17, IL-21, and IL-22 [16], also play important roles in the activity of non-specific IBD through their associated damage to the intestinal mucosa

Another important role in non-specific IBD is also played by CD40-ligands and CD40L (CD-154). CD 40/CD40L are type W2 membrane proteins similar to TNF-alpha. CD40 undergoes transient expression in activated $\mathrm{T}$ cells and is an important agent in B-lymphocyte production, as well as the activation and differentiation of monocytes and dendritic cells. Both CD and UC are characterized by local overproduction of cytokines such as TNF-alpha and an inflow of activated lymphocytes resulting in inflammation of the mucosa of the gastrointestinal tract [20]. Cytokines through their respective influence on activated T-lymphocytes and CD40 play important roles in the proliferation, differentiation, and production of immunoglobulins and in the isotype of the B lymphocytes [21-23]. The expression of CD40 on monocytes, macrophages and dendritic cells is related to the role it plays in the signalling pathway in the cellular immune response.

Adhesion of CD40 on monocytes and macrophages induces the production of pro-inflammatory cytokines and prevents apoptosis. In vivo tests have demonstrated the importance of CD40-CD40L interaction in triggering humoral immune responses, stimulating and activating specific T-cells and inducing temporary activation of macrophages. It also plays a significant part in immune responses against intracellular parasites, which are mediated by the activation of macrophages via T-lymphocytes [20]. CD40 stimulation initiates a complex, innate immune cascade which boosts the development of the systemic and local immunological pathology of the intestine. It has been found that cytokines such as TNF-alpha and IL-12 are preferentially associated with the systemic immune response; in contrast, IL-23 activity is mainly associated with the local immune response in the intestines and plays an insignificant functional role in systemic immunologic activation. It is likely that anti-CD40 application induces the activation of myeloid cells, which depends on the autocrine activation loop controlled by IL-12. Following CD40 ligation, myeloid cells produce a series of inflammatory cytokines, including IL1-12, which leads to the production of TNF-gamma by NK and other cells. This, in turn, may be repeated by the activation of myeloid cells, resulting in increased levels of pro-inflammatory factors including IL-12, TNF-alpha, MCP-1 and IL-6 [24], thus inducing inflammation of the mucosa and the deeper layers of the intestinal wall in non-specific bowel diseases.

Studies indicate that the Th9 cells producing IL-9 play a significant role in non-specific IBD, particularly UC. They develop out of naive T-lymphocytes, which transform into Th9 under the influence of IL-4 and TNF-alpha [25] Studies conducted on mouse models indicate that IL-9 is involved in triggering inflammation of the intestinal mucosa. No increase in IL-9 has been observed in the intestines of patients with $\mathrm{CD}$.

The abundance of the IL-9 receptor in the intestinal epithelial cells of patients with UC suggests that IL-9 impairs the proliferation of the intestinal epithelium and the process of healing damage resulting from chronic inflammation [26].

\section{DEVELOPMENT OF GRANULOMAS}

\section{THE PROCESS IN THE INTESTINES}

The production of IL-9 may be induced by IL-33: a cytokine which, in cases of UC, is intensively secreted by the epithelial cells of the colon under the influence of inflammatory epithelial cells $[27,28]$. The immunologic and pro-inflammatory processes occurring in the course of non-specific IBD give rise to granulomas and, in the case of $\mathrm{CD}$, non-necrotizing granulomas. They are characteristic for this condition and occur in about $40-50 \%$ of patients. They comprise epithelioid histiocytes or epithelioid cells and small amounts of multinucleated giant cells and T-lymphocytes. They are composed of epithelioid macrophages and other inflammatory cells, and an extracellular matrix surrounded by the aforementioned lymphocytes. As opposed to other granulomatous diseases, granulomas in CD are small and usually single [29].

It is believed that an important aspect of the pathophysiology of non-specific inflammatory bowel disease is the correlation between neuroendocrine peptides/amines (NEPA) of the gastrointestinal tract and the immune system [30]. The neuroendocrine regulatory system (NES) is responsible for fulfilling the major functions in the immune response of the gastrointestinal tract. The NES is composed of two parts: endocrine cells of the gastrointestinal (GI) tract in the mucosa and the enteric nervous system (ENS). GI cells are found between mucosal endothelial cells directed towards the lumen of the gastrointestinal tract and produce large amounts of hormonal peptides. Mature GI cells may produce as many as seven different hormones such as chromogranin/secretogranin, serotonin, vasoactive intestinal polypeptide (VIP), neuropeptide Y (NPY), substance P, somatostatin or ghrelin [31]. In the intestines, they play the role of immunomodulators. Of the neuropeptides, serotonin, NPY and substance P induce granulation, whereas neuropeptides, such as granin-derived peptides, VIP, somatostatin and ghrelin, reduce granulation.

Granin-derived peptides are believed to have an anti-inflammatory effects; this acts by decreasing the density of lymphocytes in inflammation sites and reducing the pro-inflammatory activity of both lymphocytes and monocytes by inhibiting the secretion of IL- 5 and IL-6 [32]. Serotonin is an important peptide secreted by mast cells, macrophages, monocytes and $\mathrm{T}$ cells, which takes part in inflammation processes in the intestines. It promotes the activation of lymphocytes, whose proliferation protects NK cells and auxiliary T cells against degradation, reduces 
immune cell (IC) apoptosis and increases T lymphocyte proliferation. Serotonin is generally considered to be a pro-inflammatory amine, as serotonin cells are capable of expressing IL-13 [30].

VIP is produced by neurons, endocrine cells and ICs. It is thought to be the main neuropeptide regulating the immune system, thus playing a significant role in inflammatory disturbances and acting as an important anti-inflammatory agent. VIP inhibits the production of pro-inflammatory cytokines such as TNF-alpha, IL-6, IL-12, iNOS and promotes the production of anti-inflammatory cytokines such as IL-10 [33]

NPY acts like hormones, neurotransmitters and neuromodulators. NPY is produced by cells such as lymphocytes, macrophages and dendritic cells during the inflammation process [34]. Substance P is a neuropeptide, a member of the tachykinine family. It is secreted by nerve fibers, i.e. intestinal efferent neurons, widely distributed on the walls of the gastrointestinal tract. It plays a significant role in the migration of intracellular ICs such as neutrophils, macrophages and T lymphocytes. Substance P is considered to be one of the main pro-inflammatory mediators in the gastrointestinal tract [30].

Somatostatin stimulates the proliferation of B-lymphoblasts and increases production of immunoglobulins; it also inhibits the activity of T-lymphocytes and granulocyte proliferation, and reduces the secretion of pro-inflammatory cytokines such as INF-gamma $[35,36]$. Ghrelin is a peptide which occurs mainly in endocrine cells of the gastric mucosa. It controls consumption of food, energy homeostasis and gastrointestinal motility. It also mediates immune responses and infections. It has an anti-inflammatory function by modulating the secretion of pro-inflammatory cytokines from stimulated macrophages [37].

\section{THE PROCESS IN THE LUNGS}

Non-necrotizing granulomas occur in various disorders such as sarcoidosis, CD common variable immunodeficiency disorders (CVID) and cryptococcosis, among others, and may also be present in tuberculosis [37]. Patients with $\mathrm{CD}$ often display a pulmonary epithelioid-like non-necrotizing granuloma with a monocyte infiltrate composed mainly of lymphocytes, plasmatic cells, neutrophils and some eosinophils [38, 39]. They occasionally present areas of central necrosis [40]. They are described as sarcoid-like granulomas since they share the same histological and immunological features and, most probably, the same development mechanism $[41,42,38]$. They comprise a central zone with CD4+ T-lymphocytes and the marginal zone with B lymphocytes and CD8+ T-lymphocytes. Non-necrotizing granulomas develop from a non-specific cellular response to unidentified antigenic factors [43] The major role in the process is played by disturbances in the number of T-reg cells. In a healthy intestine, the intestinal epithelial cells (IECs) serve as a functional barrier, while antigen-presenting cells (APCs) balance the oral immune tolerance. In non-specific IBD, this tolerance declines due to a general defect in the mucosal immune system: the balance between the functional barrier (including IEC impairment) and the innate and acquired immune response is disturbed. Such defects result in impaired communication between epithelial cells and lymphocytes, and increase disturbances between T-reg cells and CD4+T- lymphocytes, leading to chronic inflammation of the mucosa (also in the respiratory system). The intestines serve as an immunomodulator between the immune system and the epithelial cell barrier found in all the systems, as well as in the respiratory tract, which may result in the development of non-necrotizing granulomas [39]. Granulomatous processes in the lungs have a genetic basis, also common to IBD. The mutation in the NOD2 gene modifies a systemic response of the respiratory and gastrointestinal track to external factors. The NOD2 gene acts as a pattern recognition receptor (PRR) - a receptor recognizing the element of the bacterial cell wall, in particular muramyl dipeptide. This interaction is crucial for a proper response to the infection of the intestinal mucosa. A defective polymorphic variant of the gene occurs in about $15 \%$ of patients suffering from $\mathrm{CD}$ and is a risk factor for a more severe course of the disease. The same variant of the NOD2 gene is present in patients with a history of severe, chronic sarcoidosis. The significance of the aforementioned correlations becomes even more pronounced when we consider the fact that both the gastrointestinal and respiratory tracks have a common organogenetic origin, they derive from the primitive gut tube. On the basis of the presented data, the hypothesis has been fostered that this common origin of both tracks lies behind the similar lesions in these two systems in the course of IBD (Table 1) [44].

\section{RESPIRATORY TRACT COMPLICATIONS}

Extra intestinal manifestations of non-specific IBD in the lungs may cause, inter alia, diseases of the bronchi, pulmonary parenchymal diseases, thromboembolism, pleural disease, colobronchial fistula and abnormalities in pulmonary function tests. One of the symptoms of respiratory tract disease in $\mathrm{CD}$ is bronchial hyperresponsiveness, whose pathomechanism may involve subclinical inflammation of the respiratory tract triggered by several types of inflammatory cells such as macrophages or lymphocytes, as well as epithelial damage, microbleeding and mechanisms of the autonomic nervous system [49] patients with non-specific IBD and congestion of the mucous membrane, these cells and phenomena can typically be observed on bronchofiberoscopic images.

Purulent bronchitis is a commonly occurring condition manifested mainly by chronic expectoration of purulent sputum. Tracheal stenosis develops as a result of massive submucous fibrosis of inflammation lumps (granulomas) or peripheral infiltration of the mucosa. Bronchial biopsy reveals overgrowth of the mucosa, thickening of the basement membrane, angiectasis and cellular infiltrations composed of granulocytes, T-lymphocytes and B lymphocytes and non-specific chronic inflammatory infiltrations. Nevertheless, the most typical symptom observed in the 
Table I. A comparison of non-necrotizing granulomas of the lungs with those of the intestines. Own devised material based on [45-48]

\begin{tabular}{|c|c|c|}
\hline & \multicolumn{2}{|c|}{ Non-necrotizing granulomas } \\
\hline \multirow{3}{*}{ 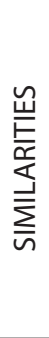 } & in the lungs & in the intestines \\
\hline & $\begin{array}{l}\text { - etiology not fully known } \\
\text { - presence of not layering epithelioid granulomas }\end{array}$ & $\begin{array}{l}\text { - both epithelia are equally prone to lesions caused by an } \\
\text { unidentified humoral factor }\end{array}$ \\
\hline & $\begin{array}{l}\text { - infiltration from lymphocytes, plasmatic cells, } \\
\text { neutrophils and some eosinophils } \\
\text { - accumulation of T CD4+ lymphocytes } \\
\text { at sites of the disease activity }\end{array}$ & $\begin{array}{l}\text { - shared embryological origin of the background for granulomas in } \\
\text { the intestines and lungs - } \\
\text { the primitive foregut, the so-called gut-lung axis } \\
\text { - the background for non-necrotizing granulomas in the intestines } \\
\text { and lungs is characterized by similar antigens }\end{array}$ \\
\hline \multirow{3}{*}{ 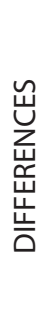 } & - enlarged regional lymph nodes & $\begin{array}{l}\text { - lymph nodes are not enlarged as intensively } \\
\text { as in the gastrointestinal tract }\end{array}$ \\
\hline & $\begin{array}{c}\text { - granulomas may develop out of } \\
\text { a background which is not affected by inflammation - } \\
\text { found on tissues where chronic inflammation process } \\
\text { does not exist }\end{array}$ & $\begin{array}{c}\text { - granulomas develop out of a background changed by } \\
\text { inflammation - found on tissues where exist chronic inflammation } \\
\text { process }\end{array}$ \\
\hline & $\begin{array}{l}\text { - histopathological image may reveal } \\
\text { a large amount of dendritic cells }\end{array}$ & - a smaller amount of dendritic cells \\
\hline
\end{tabular}

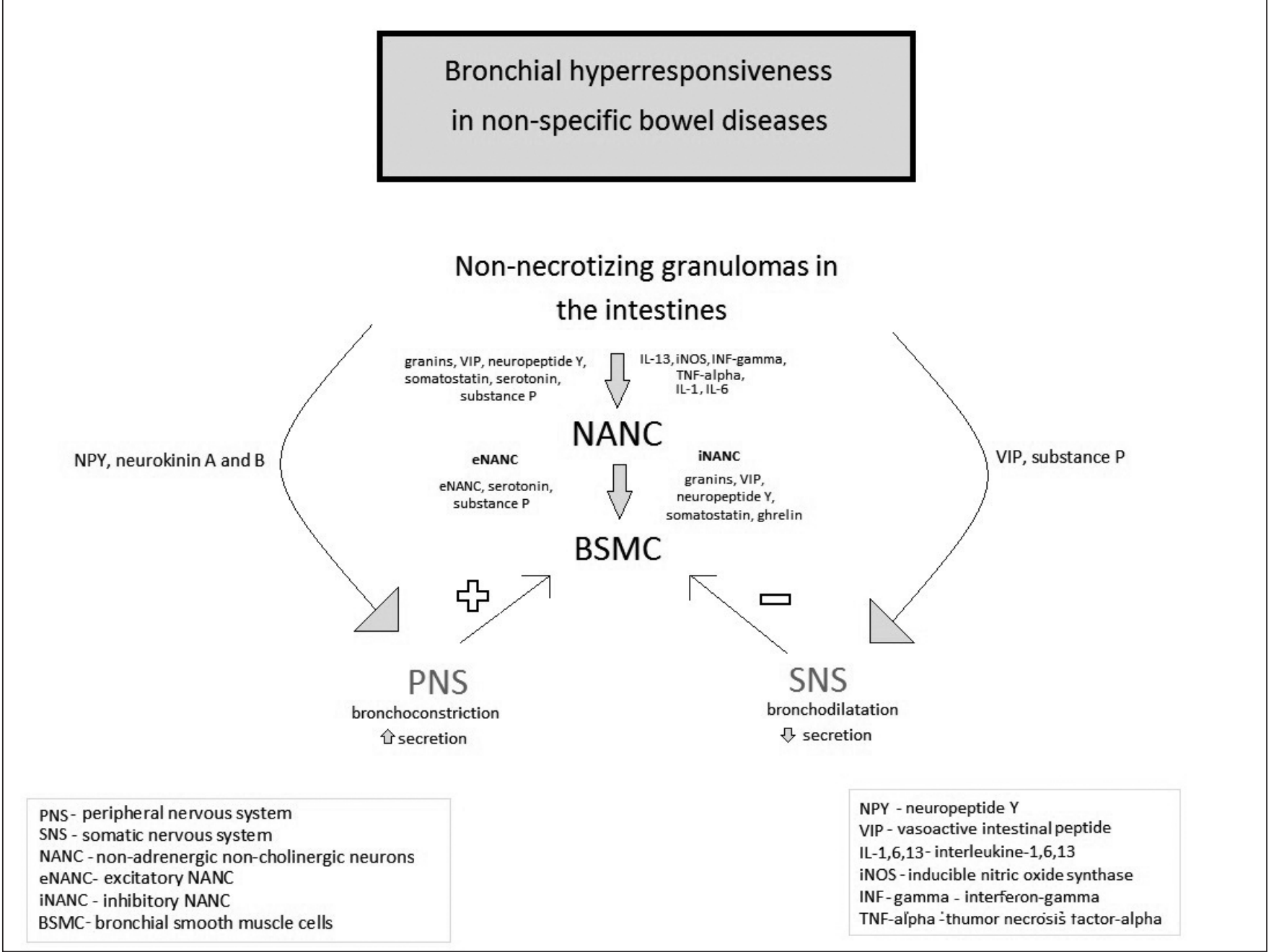

Fig. 1. The immunological mechanisms of bronchodilation and bronchoconstriction in bronchial hyperresponsiveness Based on [58]

fluid obtained by bronchoalveolar lavage (BAL) in patients with non-specific IBD is lymphocytosis. However, if a discharge is found in BAL, mostly from the trachea and bronchi, then the fluid may be dominated by neutrophils.
The most characteristic presentation of small airway disease accompanying non-specific IBD is bronchiolitis. Non-necrotizing granulomas composed of epithelial, multinucleated giant cells and dispersed mononuclear 


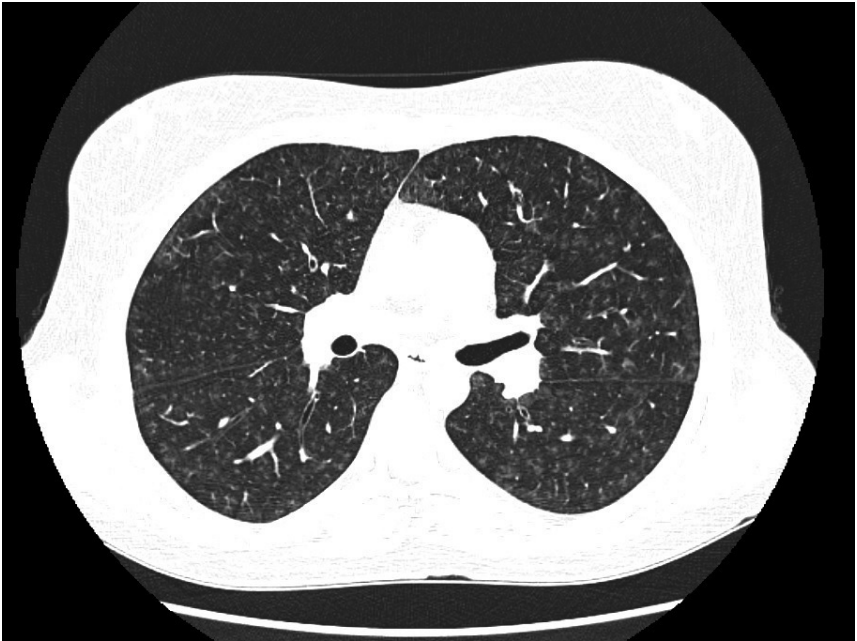

Fig. 2. CT image of lung granulomas in the form of ground glass opacities.

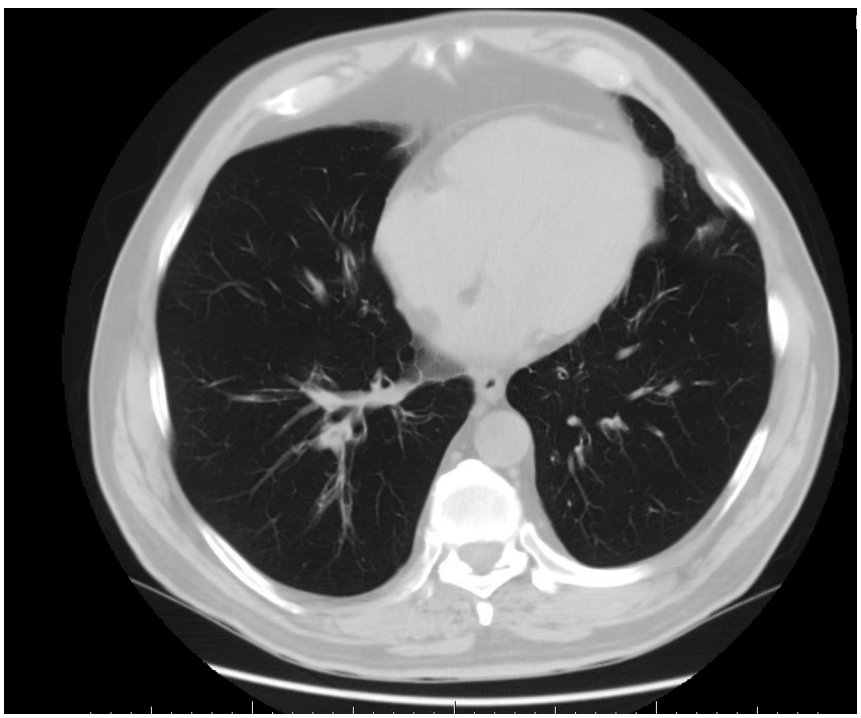

Fig. 3. HRCT image of lungs displaying non-necrotizing granulomas in the form of lumps.

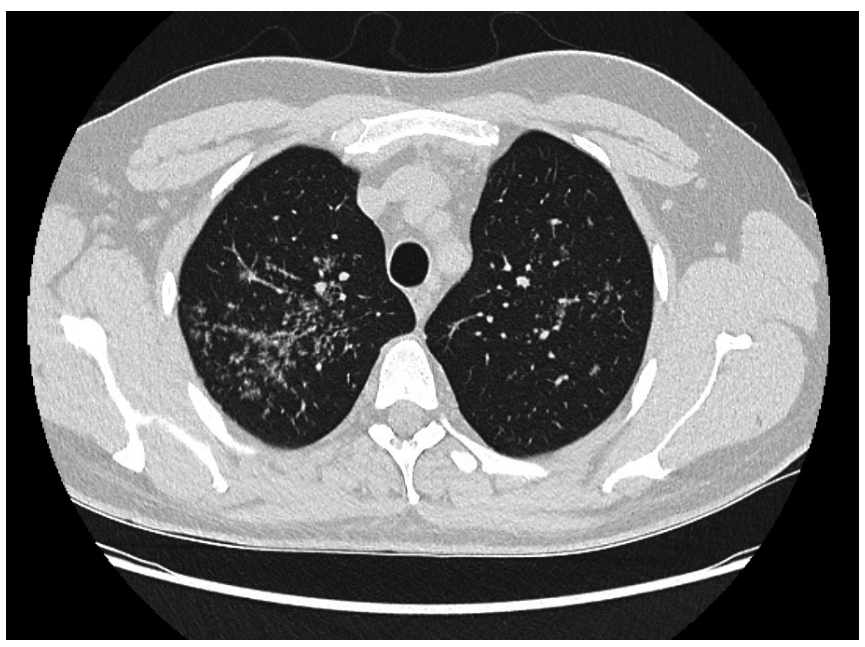

Fig. 4. CT image of lungs displaying chronic bronchitis: a thickening of the bronchial walls can be seen to the lower lobes, medial lobe, and segments of the left lung lingual.

Photographs - material from own collection cells surrounding the small bronchioles were analyzed in patients suffering from CD. Bronchiolitis related to UC is typically disperse, fibrotic and sclerosing, with a severe course, and was found to display non-granulomatous inflammation [44] For many years, studies have postulated both acute and chronic respiratory manifestations in non-specific IBD; however, the fact that they occur more rarely than in other organs contradicts the hypothesis that the lungs and intestines share the same embryological origin and that they present a similar susceptibility to immunologic diseases [50]. This contradiction may be accounted for by the fact that the epithelia of the airways contain a system that is autonomous for cells where active cilia both capture harmful substances entering the airways and initiate the defence mechanism which eliminates the harmful compound. Although the lungs also have to deal with microbiological and xenobiotic exposure, its level is not as great as that experienced by the intestinal epithelium. This may explain why the lungs are comparatively rarely affected in cases of CD [51]. Pharmacotherapy is another factor which causes further complications. Reactions such as dyspnea, coughing, impaired diffusing capacity of the lungs and interstitial pneumonia have been described in the immune complexes of the lungs during application of mesalazine, aurum salts, arechin or sulphasalazine [52, 53]. It has been demonstrated that the toxicity of sulphasalazine may mimic clinical granulation with vasculitis (Fig. 1) [54]. Sulphonamides, e.g. sulphasalazine or mesalazine, containing the residue of 5 aminosalicylic acid (5-ASA) are basic medicines used in pharmacotherapy of non-specific inflammatory bowel diseases. Drug-related adverse reactions are pulmonary complications and a characteristic radiological picture, however, their occurrence is very low. Both sulphasalazine and mesalazine can provide a picture of organizing pneumonia (OP) and their clinical signs and symptoms, as well as radiological and histopathological changes are the same as in idiopathic OP. Typical for OP peripheral interstitial radiopacities with air bronchogram of a changing location are visible on a chest X-ray. Histological examination reveals interstitial inflammation and polypoid plugs of the developing fibrous tissue filling the terminal bronchioles and parts of the alveoli. High resolution computed tomography (HRCT) depicts mainly peribronchially localized parenchymal thickening areas and small areas of ground glass opacity (Fig. 2). After using sulphasalazine for a long time, radiological features of pulmonary fibrosis similar to those found in idiopathic pulmonary fibrosis (IPF) are rarely observed. It is worth noting that new biological drugs, especially TNF-alpha antagonists (infliximab, adalimumab, etanercept) used in CD can lead to non-infectious complications including granulomatous disease and interstitial lung disease (ILD) with sub-acute or acute course resembling acute interstitial pneumonia (AIP). Occasionally, chronic eosinophilic pneumonia (CEP), OP, and diffuse alveolar haemorrhage (DAH) are also reported. The most frequently observed consequence of treatment with anti-TNF-alpha drugs, especially after using etanercept, is a mild granulomatous 
reaction which usually lasts until the end of the biological therapy and does not require any additional medical intervention. The histopathological analysis shows pulmonary alveoli with a lymphohistiocytic infiltrate, the presence of non-necrotizing granulomas and fibrosing alveolitis, with negative results of microbiological culture. In these cases, the development of ILD has a more serious prognosis and is associated with a higher risk of death. Symptoms of ILD usually appear after three infusions of TNF-alpha antagonist, however it should be mentioned that in some studies a certain number of patients (10 out of 23 ) were additionally receiving modifying antirheumatic drugs (DMARDs), including methotrexate (MTX). An important element of diagnosis in the above-mentioned field is the identification of drug-induced ILD. Its differentiation from interstitial lung diseases caused by other factors is difficult to be performed basing on clinical, radiological and histological picture (Fig. 3). There is no linear relationship between the severity of symptoms and the dose of drugs. The first symptoms usually appear only after 5-7 days of latency. The following criteria are used to recognize the drug-induced ILD:

a. Symptoms of ILD are not observed prior to initiating IBD treatment,

b. The clinical picture of drug-induced ILD should correspond to that presented in literature reports concerning drug-induced reactions,

c. Other diseases and other drugs should be excluded,

d. Symptoms of drug-induced interstitial lung disease should resolve after discontinuation of a particular drug,

e. Symptoms of drug-induced ILD recur, without any latent period, after continuing the treatment with the same drug $[44,55]$. In some patients, eosinophilia in the colon tissue has been found to be higher in subjects with positive tests for oral allergens, which may indicate that atopy may be a common basis for the occurrence of pulmonary lesions in the course of IBD. In epidemiological studies, a high correlation between people suffering from chronic obstructive pulmonary disease (COPD) and those with IBD has been demonstrated. The risk of COPD development in patients with CD increases about 2.7 times, and 1.8 in patients with UC, as compared to healthy people [56].

In various studies, attention has been paid to the relationship between severity of IBD and lesions in the airways. In some of them, patients reported a greater number of respiratory symptoms during exacerbations of the disease course, and observed less symptoms when bowel disease subsided. An increased number of respiratory symptoms associated with a high endoscopic activity index value was indicated by patients with UC, however no correlation between the actual IBD activity and pulmonary lesions was detected. Changes in the pulmonary function test (PFT) were more numerous in patients with active IBD. Spirometry findings showed lower levels of FEV1, FVC, FEV1/VC in patients with IBD exacerbations and an elevated level of RV, RV/TLC between remissions. No relationship between the duration of IBD and the result of PFT as well as between the results of the bronchial hyperresponsiveness test with the use of metacholine and the course of inflammatory bowel diseases was observed. Although some researchers have pointed to the possible correlation between the duration of IBD, and HRCT findings, no relationship was finally stated between them. The analysis of numerous clinical cases shows a higher number of respiratory complications with an increase in the duration of IBD exacerbations [57]. It should also be noted that although the systemic lesions in the respiratory system can occur in any age, the female gender is more predisposed. The most common symptom of airway inflammation is a cough with a profuse expectoration of purulent sputum. Lesions in the larynx and trachea can give hoarseness and stridor. Sometimes the bronchoscopic examination reveals the nodules of an inflammatory nature, which can to a large extent narrow the airways. If left untreated, they can lead to tracheal stenosis, bronchiectasis, deformities, and even fistulas. In these cases, clinical and histopathological picture presents granulomatous, necrotizing, purulent or obstructive bronchiolitis. Recent studies indicate an increased risk of asthma in UC and CD patients. The most common form of lesions, besides those in the airways and pleura, is the organizing pneumonia $(\mathrm{OP})$ usually located in the upper regions of the lungs, but it can also involve the entire lungs and even lead to respiratory failure. Other types of ILD in the course of IBD are non-specific interstitial pneumonia (NSIP), desquamative interstitial pneumonia (DIP), and pulmonary interstitial emphysema (PIE). In BAL, a high percentage of lymphocytes can be found in $\mathrm{CD}$ patients and their characteristics may be identical to that of sarcoidosis. In differential diagnosis, it is useful to assess serum angiotensin-converting enzyme (ACE) activity (high in sarcoidosis, low in CD). Small, sterile, necrotizing nodules, which may undergo breakdown, have also been observed in the pulmonary interstitium of IBD patients. They are usually accompanied by necrotic bronchitis, which should be differentiated from granulomatosis with polyangitis (GPA) (Fig. 4) [55].

\section{CONCLUSIONS}

Evidence suggests that $\mathrm{CD}$ and UC, described as non-specific IBD characterized by varied symptoms in a number of systems, are not so much inflammatory conditions affecting the intestines, but rather a group of multisystem inflammatory diseases, in which all manifestations observed in other systems, such as the respiratory tract, are coexisting symptoms. The clinical symptoms presented by patients are manifested as an effect of the chronic inflammation representing the underlying cause of the condition. The key role in disease initiation is played by acute phase proteins such as IL-6, IL-12, IL-21, IL-23, TNF-alpha and INF-gamma; an important part is also played by the activation of membrane proteins such as CD40/CD40L and the general destabilization of immune system function, for example, inflammation of the mucous membranes. Additionally, immune system function is further disturbed 
by the action of neuroendocrine peptides/amines. The pathomechanisms analyzed in this article manifest as different symptoms in various parts of the body, for example, where inflammation may be manifested by the presence of non-necrotizing granulomas in the respiratory tract. The granulomas occurring in the intestines show a number of histopathological and immunological similarities to those found in the airways. This similarity hints at the presence of an underlying connection between co-occurring diseases in different systems. It also highlights their destructive nature, manifested in the intestines as abscesses, ulcers, fistulas or post-inflammatory adhesions, and in the lungs as restriction resulting from pulmonary parenchymal disease, bronchitis, pleuritis and colobronchial fistulas; their occurrence is also associated with an increased risk of thromboembolism, coughing and dyspnea.

\section{REFERENCES}

1. Bartnik W, Strzeszyński $Ł$, Szczepanek M. Wrzodziejące zapalenie jelita grubego. In:Gajewski P, Niżankowska-Mogilnicka E, Mejza F (eds). Interna Szczeklika. Medycyna Kraków: Praktyczna, 2017, pp. 1021-1028

2. Bartnik W, Strzeszyński Ł, Szczepanek M. Choroba Leśniowskiego i Crohna. In: Gajewski P, Niżankowska-Mogilnicka E, Mejza F (eds.). Interna Szczeklika. Kraków: MedycynaPraktyczna, 2017 pp. 1028-1034.

3. Feuerstein JD, Cheifetz AS. Ulcerative Colitis. Mayo Clin Proc. 2014;89(11):1553-1563

4. Wiercinska-Drapalo A, Jaroszewicz J, Flisiak R, et al.Epidemiological characteristics of inflammatory bowel disease in North-Eastern Poland. World J Gastroenterol. 2005;11(17):2630-2636.

5. M'Koma AE. Inflammatory bowel disease. An expanding global health problem. Clin Med Insights Gastroenterol. 2013;14;6:33-47.

6. Rydzewska GM, Głuszek-Osuch M, Kawalec P, et al. Raport społeczny. Nieswoiste zapalne choroby jelit - przeciwnik rosnący w siłę.Warszawa, 2016;

7. Jakubowski A, Zagórowicz E, Kraszewska E, et al. Rising hospitalization rates for inflammatory bowel disease in Poland. Pol Arch Med Wewn. 2014;124(4):180-90

8. Jacob P. Epidemiology and risk factors. Chronic Venous Disord Low Limbs A Surg Approach. 2015;12(4):35-39.

9. Betteridge JD, Armbruster SP, Maydonovitch C, et al. Inflammatory bowel disease prevalence by age, gender, race, and geographic location in the U.S. military health care population. Inflamm Bowel Dis. 2013;19(7):1421-1427

10. Li G, Ren J, Li J. .Susceptibility genes and serum markers of intestinal fibrosis in Crohn disease. Zhonghua Wei Chang Wai Ke Za Zhi. 2017; 20(11):1326-1330.

11. Neuman MG, Nanau RM. Inflammatory bowel disease: role of diet, microbiota, life style. Translat Res. 2012;160(1):29-44

12. Negroni A, Pierdomenico M, Cucchiara S, et al. NOD2 and inflammation: Current insights. J Inflamm Res. 2018;11:49-60

13. Xu XR, Liu CQ, Feng BS, et al. Dysregulation of mucosal immune response in pathogenesis of inflammatory bowel disease. World J Gastroenterol. 2014; 20(12): 3255-3264

14. Mannon PJ, Fuss IJ, Mayer L, et al. Anti-Interleukin-12 Antibody for Active Crohn's Disease. N Engl J Med. 2004;351(20):2069-2079

15. Hue S, Ahern P, Buonocore $S$, et al. Interleukin-23 drives innate and T cellmediated intestinal inflammation. J Exp Med. 2006;203(11):2473-2483

16. Strober W, Fuss IJ. Pro-inflammatory cytokines in the pathogenesis of inflammatory bowel diseases. Gastroenterology. 2011;140(6):1756-1767.
17. Korn T, Bettelli E, Gao W, et al. IL-21 initiates an alternative pathway to induce pro-inflammatory TH17 cells. Nature. 2007 ;448(7152):484-487

18. LiuZ, Feng BS, Yang SB, Chen X, Su J, Yang PC. Interleukin (IL)-23 suppresses IL-10 in inflammatory bowel disease. J Biol Chem. 2012;287(5):35913597

19. Hundorfean G, Neurath MF, Mudter J. Functional Relevance of T Helper 17 ( Th17) Cells and the IL-17 Cytokine Family in Inflammatory Bowel Disease. Inflamm Bowel Dis.2012;18(1):180-186.

20. LiuZ, ColpaertS, D'Haens GR, et al. Hyperexpression of CD40 ligand (CD154) in inflammatory bowel disease and its contribution to pathogenic cytokine production. J Immunol. 1999;163:4049-4057.

21. Banchereau J, Bazan F, Blanchard D, et al.The CD40 antigen and its ligand. Annu Rev Immunol. 1994; 12:881-922.

22. Foy TM, Aruffo A, BajorathJ.Foy, et al. Immune regulation by CD40 and its ligand gp39. Annu.Rev.Immunol.1996;14:591-617.

23. van Kooten C,BanchereauJ, Functions of CD40 on B cells, dendritic cells and other cells. Curr Opin Immunol. 1997;9(3):330-337.

24. Uhlig HH, McKenzie BS, Hue S, et al. Differential Activity of IL-12 and IL-23 in Mucosal and Systemic Innate Immune Pathology. Immunity. 2006;25(2):309-18.

25. Veldhoen M, Uyttenhove C, van Snick J, et al. Transforming growth factorbeta reprograms the differentiation of $T$ helper 2 cells and promotes an interleukin-9-producing subset. Nat Immu- nol. 2008;9:1341-1346.

26. Giuffrida P, Corazza GR, Di Sabatino A. Old and New Lymphocyte Players in Inflammatory Bowel Disease. Dig Dis Sci. 2018;63(2):277-88.

27. Blom L, Poulsen BC, Jensen BM, et al. IL-33 induces IL-9 production in human CD4+ T cells and basophils. PLoS One. 2011;6(7)e21695.

28. Seidelin JB, Bjerrum JT, Coskun M, et al. IL-33 is upregulated in colonocytes of ulcerative colitis. Immunol Lett. 2010;128:80-85

29. Bartnik W, Wejman J. Atlas kliniczno-patologiczny nieswoistych chorób zapalnych jelit. Poznań: Termedia Wydawnictwa Medyczne, 2011, p. 75.

30. El-Salhy M, Solomon T, Hausken T, et al. Gastrointestinal neuroendocrine peptides/amines in inflammatory bowel Disease. World J Gastroenterol. 2017;23(28):5068-5085.

31. Egerod KL, Engelstoft MS, Grunddal KV, et al. A major lineage of enteroendocrine cells coexpress CCK, secretin, GIP, GLP-1, PYY, and neurotensin but not somatostatin. Endocrinology 2012;153:5782-5795

32. Deftos LJ. Chromogranin A: its role in endocrine function and as an endocrine and neuroendocrine tumor marker. Endocr Rev. 1991;12:181187

33. Ganea D, Hooper KM, Kong W. The neuropeptide vasoactive intestinal peptide: direct effects onimmune cells and involvement in inflammatory and autoimmune diseases. Acta Physiol (0xf). 2015;213:442-452

34. Wheway J, Mackay CR, Newton RA, et al.Fundamental bimodal role for neuropeptide $Y 1$ receptor in the immune system. J Exp Med. 2005;202:1527-1538

35. RosskopfD, Schürks M, Mantheyl, et al. Signaltransduction of somatostatin in human B lymphoblasts. Am J Physiol Cell Physiol 2003;284:179-190

36. Penman E, Wass JA, Butler MG, et al. Distribution and characterisation of immunoreactive somatostatin in human gastrointestinal tract. Regul Pept 1983;7:53-65.

37. Waseem $\mathrm{T}$, Duxbury $\mathrm{M}$, Ito $\mathrm{H}$, et al. Exogenous ghrelin modulates release of pro-inflammatory and antiinflammatory cytokines in LPSstimulated macrophages through distinct signaling pathways. Surgery 2008; 143:334-342.

38. Levenbrown Y, Tauber D, Hall OR, et al. Granulomatous lung disease as the initial presentation of crohn disease. J Pediatr Gastroenterol Nutr. 2009;48(4):487-90. 
39. Sartini A, Bianchini M, Schepis F, et al. Complete resolution of nonnecrotizing lung granuloma and pyoderma gangrenosum after restorative proctocolectomy in a woman with severe ulcerative colitis and cytomegalovirus infection. Clin Case Reports. 2016;4(2):195-202.

40. Majewski S, Piotrowski W. Pulmonary manifestations of inflammatory bowel disease. Arch Med Sci. 2015;11(6):1179-88.

41. Takeda S, Akagi T, Miyazaki H, et al. Two patients with new granulomatous lung lesions during treatment of Crohn's disease. Respir Med Case Reports. 2014;12:16-18.

42. Vandenplas 0, Casel S, Delos M, et al. Granulomatous bronchiolitis associated with Crohn's disease. Am J Respir Crit Care Med. 1998;158(5 PART I):1676-1679.

43. Ma YL, Gal A, Koss M. Reprint of:The pathology of pulmonary sarcoidosis: update. Semin Diagn Pathol. 2018;35(5):324-333.

44. Majewski S, Piotrowski W. Pulmonary manifestations of inflammatory bowel disease. Arch Med Sci. 2015;11(6):1179-88.

45. Bernstein CN, Fried M, Krabshuis JH, et al. World gastroenterology organization practice guidelines for the diagnosis and management of IBD in 2010. Inflamm Bowel Dis. 2010;16(1):112-24

46. Puntis JWL, Tarlow MJ, RaafatF, et al. Crohn's disease of the lung. Arch Dis Child. 1990;65(11):1270-1271

47. Vandenplas 0 , CaselS, Delos M, etal.Granulomatous bronchiolitis associated with Crohn's disease. Am JRespir CritCare Med. 1998;158(5p.l):1676-1679

48. Bargagli E, Rottoli P, Torricelli E, et al. Airway-Centered Pleuroparenchymal Fibroelastosis Associated with Non-Necrotizing Granulomas: A Rare New Entity. Pathobiology. 2018;85(5-6):276-79

49. Mansi A, Cucchiara S, Greco L, et al. Bronchial hyperresponsiveness in children and adolescents with Crohn's disease. Am J Respir Crit Care Med. 2000;161(3 I):1051-1054.

50. Rodriguez-Roisin R, Bartolome SD, Huchon G, et al. Inflammatory bowel diseases, chronic liver diseases and the lung. EurRespir J.2016;47(2):638-650.

51. Lu DG, Ji XQ, Liu X, et al. Pulmonary manifestations of Crohn's disease. World J Gastroenterol. 2014;20(1):133-141.

52. Jones GR, Malone DN. Sulphasalazine induced lung disease. Thorax. 1972;27(6):713-717.

53. Davies D, Macfarlane A. Fibrosing alveolitis and treatment with sulphasalazine. Gut. 74; 15(3):185-188.

54. Salerno SM, Ormseth EJ, Roth BJ, et al. Sulfasalazine pulmonary toxicity in ulcerative colitis mimicking clinical features of Wegener's granulomatosis. Chest. 1996;110(2):556-559
55. Wiatr E, Rowińska-Zakrzewska E, Pirożyński M. Choroby śródmiąższowe płuc. Warszawa: Alfa-Medica Press, 2012.

56. Ekbom A, Brandt $\mathbb{E L}$, Granath $Æ F$, et I. Increased Risk of Both Ulcerative Colitis and Crohn 's Disease in a Population Suffering from COPD. Lung. 2008; 186: 167-72

57. Kacprzak A, Szturmowicz, Kuś J. Respiratory system involvement in inflammatory bowel diseases. Adv. Respir. Med. 2017;85: 161-168

58. CMKP. Specialization course of pulmonary diseases. Olsztyn, 2014.

\section{ORCID and contributionship:}

Igor Rakoczy-0000-0001-7748-8890 A,B,C,D,E,F

Marzena Ocetek - 0000-0002-3876-292X B,C,D

Marzena Wiatr - 0000-0001-5854-1078 B,C,D

Patrycja Zięba - 0000-0002-2064-9873 B,C,D

Michat Panek - 0000-0002-3718-4793 B,C,D,E

Piotr Kuna - 0000-0003-2401-0070 ${ }^{E}$

\section{Conflict of interest}

Authors declare no conflict of interest

\section{CORRESPONDING AUTHOR Igor Rakoczy}

Department of Internal Medicine, Asthma and Allergy,

Medical University of Lodz, Lodz, Poland

ul. Kopcinskiego 22, 90-153 Lodz, Poland

e-mail: igor_rakoczy@02.pl

Received: 12.02 .2020

Accepted: 29.06 .2020

A - Work concept and design, B - Data collection and analysis, C - Responsibility for statistical analysis,

D-Writing the article, $\mathbf{E}$-Critical review, $\mathbf{F}$ - Final approval of the article 